\title{
MAPEAMENTO DA SECAGEM MICRO-ONDAS EM CASCALHOS CONTAMINADOS COM FLUIDO DE PERFURAÇÃO
}

\author{
N. CARNEIRO, I. J. PETRI e C. H. ATAIDE \\ Universidade Federal de Uberlândia, Faculdade de Engenharia Química \\ E-mail para contato: chataide@feq.ufu.br
}

\begin{abstract}
RESUMO - A perfuração de poços de petróleo geram fragmentos de rochas, também chamados cascalhos, que estão contaminados com fluido de perfuração, que é injetado para a uma perfuração mais eficiente. Este cascalho contaminado precisa de um devido tratamento para que ocorra seu descarte em alto mar. Um equipamento muito utilizado nesse tratamento é a centrifuga vertical, porém ela opera muito próximo dos limites imposto pela legislação ambiental que estipula um teor de $6,9 \%$, em massa, de contaminante no cascalho. Portanto, o equipamento de descontaminação de cascalhos de perfuração que tem sido proposto pelos autores desse trabalho é um secador via micro-ondas. Foram realizadas secagens de um cascalho de perfuração contaminado com $24,4 \%$ de fluido de perfuração BRMUL base nparafina. Utilizaram-se energias específicas de $0,200,0,230$ e $0,260 \mathrm{kWh} / \mathrm{kg}$ e uma temperatura de controle de $290^{\circ} \mathrm{C}$. Após a secagem foi feito um mapeamento de cada região do recipiente e analisado, pelo método da retorta, os teores residuais presentes em cada região. Constataram-se a influencia de alguns fatores que causam a heterogeneidade da secagem como, a existência de um gradiente de temperatura no equipamento devido aos hotspots, a localização do termopar no interior da cavidade e o tempo de secagem. Os resultados mostram que regiões próximas às bordas da bandeja apresentam um alto teor residual, regiões próximas ao termopar apresentam baixo teor residual.
\end{abstract}

\section{INTRODUÇÃO}

Para se perfurar um poço de petróleo utiliza-se o método de perfuração rotativo. Este método baseia-se em pressionar a broca sobre a rocha e realizar um movimento de rotação ocasionando o esmerilhamento da rocha. A retirada destes fragmentos de rocha gerados, ditos cascalhos de perfuração, é realizada pelo fluido de perfuração, que entra pelo interior da broca e retorna à superfície, pelo espaço anular entre a coluna de perfuração e as paredes do poço, carreando consigo os cascalhos.

No intuito de descontaminar o cascalho da perfuração de poços e de recuperar o fluido de perfuração para sua reutilização, várias etapas de separação são necessárias. Cada equipamento desse sistema é responsável pela separação de determinada faixa de tamanho de partículas e pela diminuição dos teores de fluido (ou base sintética do fluido) do cascalho contaminado. Para adequar o cascalho aos limites ambientais previstos é empregado o "secador de cascalho" que, na verdade, é uma centrífuga filtrante. Porém, devido aos riscos operacionais e também ao fato do teor residual de fluido presente no cascalho estar muito próximo dos limites ambientais, bem como pela provável redução destes limites, há uma busca por novas tecnologias de descontaminação de cascalhos. 
Uma tecnologia eficaz que vem sendo citada na literatura para descontaminação de cascalho é a secagem por micro-ondas. Os estudos de descontaminação de cascalhos contaminados com fluido de perfuração de base não-aquosa, utilizando as micro-ondas iniciaram com Shang et al. (2005, 2006, 2007), Robinson et al. (2009, 2010, 2012) e passaram a ser estudados mais recentemente por Pereira (2013), Santos (2014), Panisset (2014) e Petri (2014). Os resultados reportados na literatura mostram que esta tecnologia tem potencial para substituir a centrífuga filtrante, reduzindo os teores de fase orgânica a níveis abaixo de $1 \%$, em massa.

Mesmo que esse tipo de equipamento tenha sido estudado por quase uma década, ainda existem vários desafios a serem vencidos. Muitos parâmetros e condições operacionais devem ser ainda estudados para que essa tecnologia avance e possa ser utilizada industrialmente na descontaminação de cascalhos de perfuração.

Por essa razão este trabalho tem como objetivo mapear a secagem de cascalhos de perfuração utilizando um equipamento de secagem micro-ondas batelada escala de bancada. Para isso, foram recolhidas amostras de várias regiões do recipiente de secagem e analisados os teores residuais de fase orgânica presente nessas regiões. Através destes resultados foi possível verificar a influência dos hotspots formado pelas ondas eletromagnéticas e por outros fatores como: borda da bandeja, exaustor, termopar.

\section{MATERIAIS E MÉTODOS}

\subsection{Unidade Experimental}

Todos os experimentos de secagem foram realizados utilizando um micro-ondas comercial da marca SHARP ${ }^{\circledR}$ modelo R-23GT com $1600 \mathrm{~W}$ de potência, frequência de 2450 $\mathrm{MHz}$, cavidade em aço inox com dimensões internas de 330x330x181 mm. Esse equipamento não apresenta prato giratório, mas possui um disco giratório situado na parte superior da cavidade que é capaz de distribuir melhor as micro-ondas no seu interior. O forno é equipado com dois magnétrons e duas antenas, uma situada na parte superior e a outra na parte inferior da cavidade. O aparato conta também com um sistema de exaustão, que se situa na parte superior esquerda da cavidade e os gases oriundos do processo de secagem via micro-ondas passam por um mini sistema de chicanas até sairem para o exterior do equipamento.

Foram feitas algumas adaptações no equipamento para aferição de temperaturas e pressão, assim como injeção de gás inerte $\left(\mathrm{N}_{2}\right)$ e ar comprimido. A Figura 1 mostra a unidade de secagem com todas as adaptações feitas. Sendo: (1) manômetro transmissor de pressão; (2) anemômetro de fio quente; (3) sistema de válvulas de alimentação de gás inerte $\left(\mathrm{N}_{2}\right)$; (4) termopar tipo K para aferição de temperaturas do vapor; (5) termopar tipo K para aferição de temperaturas do leito; (6) disco giratório original do equipamento para melhor distribuição das micro-ondas; (7) - recipiente de borosilicato onde o material é alocado.

Figura 1 - Unidade experimental de secagem micro-ondas 

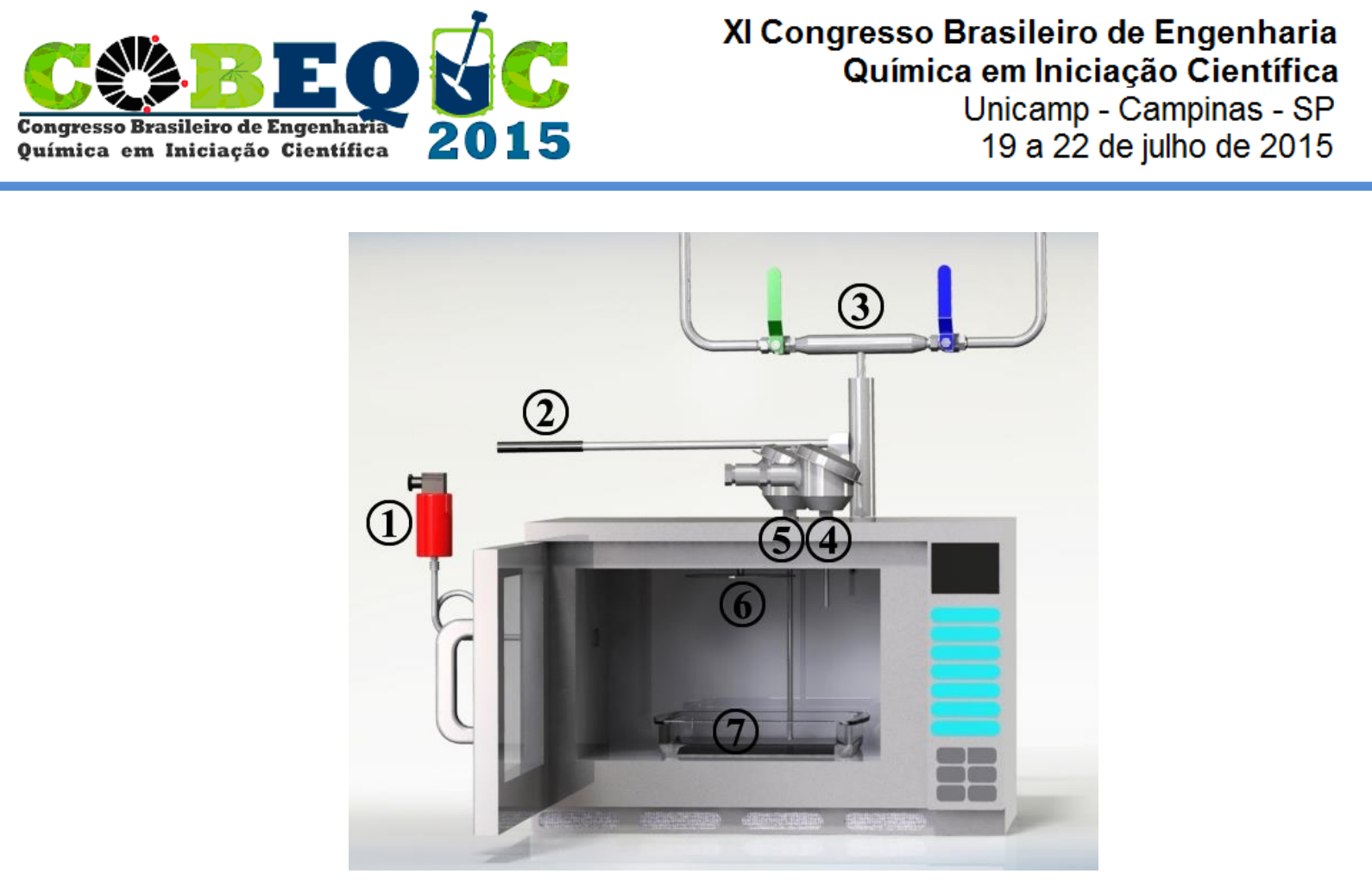

\subsection{Materiais e procedimento experimental}

Os testes de secagem em micro-ondas foram realizados com cascalhos oriundos do processo da perfuração de poços de petróleo e doados pela empresa Petrobras S.A. Esse cascalho foi advindo da Central de Cascalhos da Petrobras situada em Carmópolis-SE.

A contaminação do cascalho foi feita empregando-se fluido de perfuração BRMUL base n-parafina, o qual foi doado pela Petrobras S.A.. Essa escolha de utilizar o fluido de perfuração foi feita visando obter um cascalho com um maior teor de contaminantes.

Para cada experimento eram feitas análises de retorta para obter o teor de n-parafina e água presentes no cascalho. As retortas foram feitas utilizando um Kit da marca FANN com $50 \mathrm{~mL}$ de capacidade. O procedimento se iniciava pesando a amostra de material ou fluido que era, posteriormente, adicionado a uma célula de aquecimento que atingia temperaturas de $400^{\circ} \mathrm{C} \pm 40^{\circ} \mathrm{C}$, com isso os componentes voláteis eram evaporados. Os vapores passavam através de um condensador e eram coletados em uma proveta. Através de um balanço de massa determinava-se o teor de fase orgânica e fase aquosa.

Os ensaios de secagem foram realizados utilizando um cascalho contaminado com $24,4 \%$ de fluido (12,6\% de n-parafina inicial), submetido a três energias específicas diferentes: $0,20,0,23$ e $0,26 \mathrm{kWh} / \mathrm{kg}$. A temperatura de controle foi $290^{\circ} \mathrm{C}$ e a vazão de inerte foi $70 \mathrm{~L} / \mathrm{min}$.

Após a secagem, o recipiente com o cascalho era resfriado à temperatura ambiente e o leito era seccionado em várias partes, conforme tonalidade de cor da superfície do material. Então, pelo menos $50 \mathrm{~mL}$ desse material era retirado e submetido ao teste de retorta para determinar o teor de n-parafina na região correspondente. Então foi esquematizado uma representação das regiões das amostras com seus respectivos teores de n-parafina. Depois de obtidos os resultados dos mapeamentos descritos anteriormente, foram realizados os mesmos ensaios de secagem, mas ao fim do experimento todo o material do recipiente foi homogeneizado e foi feita a análise de teor médio de n-parafina residual. 


\section{RESULTADOS E DISCUSSÕES}

Os resultados dos mapeamentos dos teores de n-parafina para uma energia específica de $0,200,0,230,0,260 \mathrm{kWh} / \mathrm{kg}$ são mostrados na Figura 2, 3 e 4, respectivamente. Cada resultado contém três imagens: a primeira é a foto real do material após a secagem, a segunda é o mapeamento visual das regiões e seus respectivos teores de n-parafina residual, a terceira é o teor de n-parafina do cascalho homogeneizado. Os resultados mostram que existe um gradiente de temperatura no equipamento, pois há regiões do recipiente em que há um menor teor residual de n-parafina do que outros.

Figura 2 - Mapeamento do teor de n-parafina no recipiente a $0,200 \mathrm{kWh} / \mathrm{kg}$
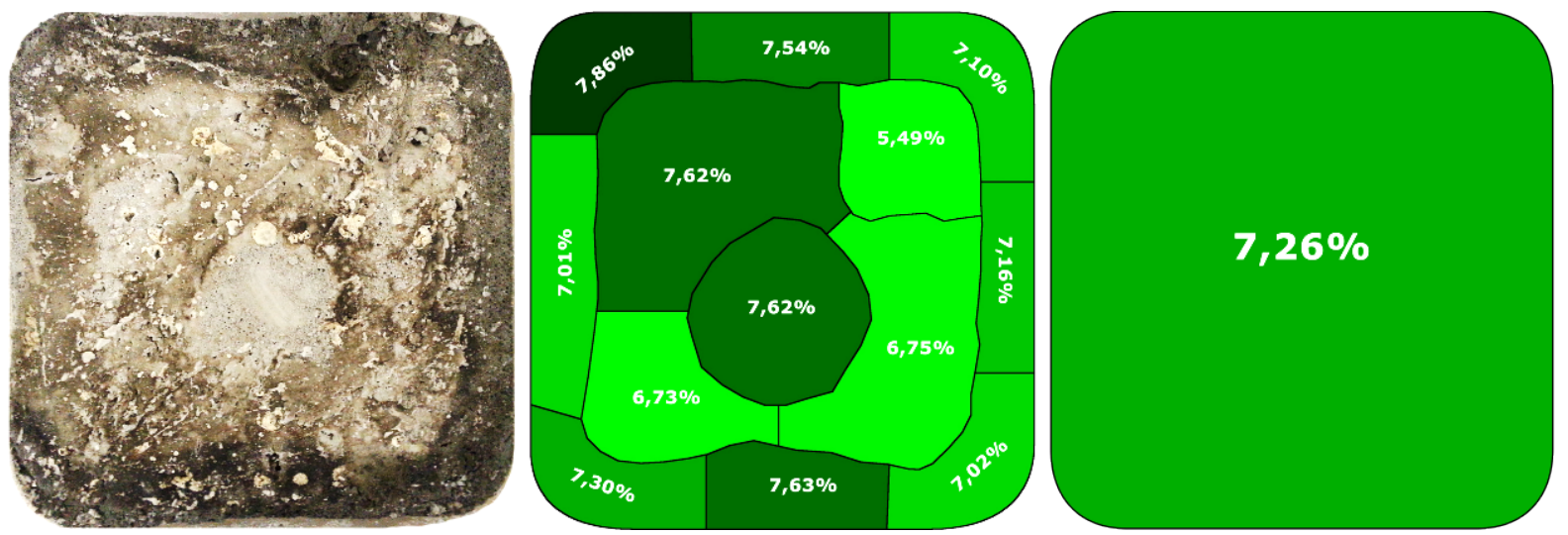

Para a Figura 2, a região de menor teor residual $(5,49 \%)$ é a região onde fica localizado o termopar que mede a temperatura do leito. Nessa região, o metal do termopar faz com que as micro-ondas sejam refletidas aumentando a incidência de micro-ondas no local. Já o centro do recipiente, regiões com teores de 7,62\%,6,73\% e 6,75\% de n-parafina residual, são locais que percebe a presença de regiões heterogêneas de secagem devido, possivelmente, ao gradiente de temperatura no interior do equipamento. $\mathrm{O}$ restante das regiões é influenciado pelas bordas do recipiente. Ao se homogeneizar o material, o teor de n-parafina residual é de $7,26 \%$, constatando uma grande diferença entre o teor de algumas regiões do recipiente com o teor do cascalho homogeneizado.

Figura 3 - Mapeamento do teor de n-parafina no recipiente a $0,230 \mathrm{kWh} / \mathrm{kg}$
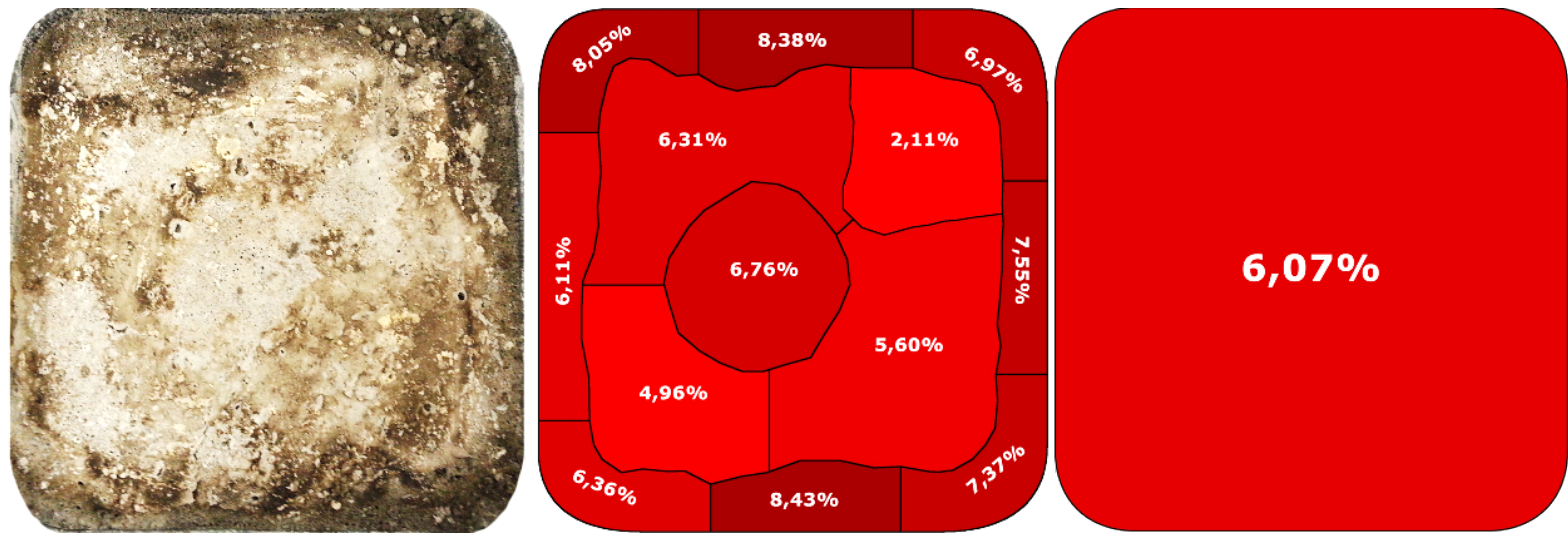
A Figura 3 mostra que o ponto com menor teor de n-parafina residual $(2,11 \%)$ é também onde fica localizado o termopar que mede a temperatura do leito. Há a presença de gradientes de temperatura mostradas nas regiões com teores de 6,31\%,6,76\%, 4,96\% e 5,60\% de n-parafina residual (centro do recipiente), como constatado também na Figura 2. Nesse ensaio de secagem, foi possível perceber mais precisamente a influência das bordas do recipiente. Nestas regiões ocorre, possivelmente, a condensação da n-parafina já evaporada, motivo pelo qual foram observados os maiores teores residuais. $\mathrm{O}$ teor de n-parafina residual do cascalho homogeneizado foi $6,07 \%$, que difere bastante de algumas regiões do mapeamento.

Figura 4 - Mapeamento do teor de n-parafina no recipiente a $0,260 \mathrm{kWh} / \mathrm{kg}$
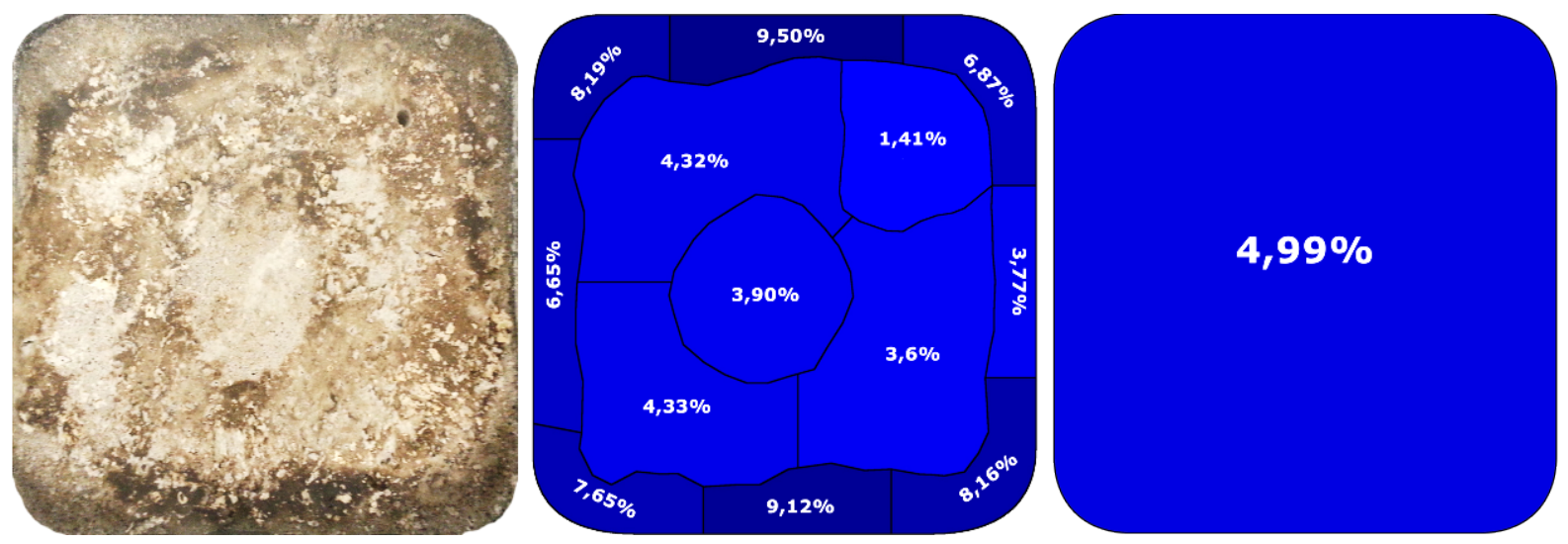

Na Figura 4 verifica-se que a influência do termopar que mede a temperatura do leito é bem nítido, chegando a teores próximo de $1 \%$ enquanto o teor de n-parafina residual do material homogeneizado está em 4,99\%. Nesse caso, percebe-se que o gradiente de temperatura das regiões com 4,32\%, 4,33\%, 3,6\% e 3,9\% de n-parafina residual não está tão acentuado como os anteriores, mas ainda há um gradiente visível. A influência das bordas do recipiente é bem pronunciada, o que mostra que quanto mais tempo o material é deixado no interior do equipamento, menores os gradientes no centro do recipiente. A região de 3,77\% não houve efeito de borda do recipiente, pois provavelmente a influência do gradiente de temperatura foi mais acentuado, secando mais essa região.

\section{CONCLUSÕES}

A operação de secagem micro-ondas na descontaminação de cascalhos de perfuração se mostrou eficaz, reduzindo os teores de n-parafina de $12,6 \%$ para $4,99 \%$, em média, para uma energia específica relativamente baixa $(0,260 \mathrm{kWh} / \mathrm{kg})$

Conclui-se também que um secador micro-ondas multi-mode pode apresentar um gradiente de temperatura em sua cavidade, fazendo com que o material seja secado de maneira heterogênea. A presença de um metal no interior da amostra (termopar) faz com que aumente a incidência de micro-ondas em suas proximidades e com isso haja uma maior secagem naquele local. As bordas do recipiente em que se armazena o material para a secagem faz com que haja condensação de vapores, o que aumenta os teores de n-parafina em regiões adjacentes a esta borda. 


\section{REFERÊNCIAS}

PANISSET, C. M. A., Novas alternativas para o sistema de separação sólido-líquido na perfuração de poços de petróleo: teste de uma unidade de VCS; busca do aumento de eficiência da operação de hidrociclonagem; e tratamento da borra de centrifugação por microondas, Uberlândia/MG, UFU, 2014.

PEREIRA, M. S., Aplicação de secagem por micro-ondas no tratamento de cascalho de perfuração, Uberlândia/MG, UFU, 2013.

PETRI, I. J., Secagem por micro-ondas na descontaminação de cascalhos de perfuração, Uberlândia/MG, UFU, 2013.

ROBINSON, J. P., BINNER, E. R., SILVESSER, S. A., KINGMAN, S. W., LESSER, E. H., Investigation into the mechanisms by which microwave heating enhances separation of water-in-oil emulsions, Fuel, v. 116, p. 516-521, 2014.

ROBINSON, J. P., KINGMAN, S. W., LESSER, E. H., YI, C., Microwave remediation of hydrocarbon-contaminated soils - Scale-up using batch reactors, Separation and Purification Technology, v. 96, p. 12-19, 2012.

ROBINSON, J. P., KINGMAN, S. W., SNAPE, C. E., BARRANCO, R., SHANG, H., BRADLEY, M. S. A., BRADSHAW, S. M., Remediation of oil-contaminated drill cuttings using continuous microwave heating, Chemical Engineering Journal, v. 152(2), p. 458-463, 2009.

ROBINSON, J. P., KINGMAN, S. W., SNAPE, C. E., BRADSHAW, S. M., BRADLEY, M. S. A., SHANG, H., BARRANCO, R., Scale-up and design of a continuous microwave treatment system for the processing of oil-contaminated drill cuttings, Chemical Engineering Research and Design, v. 88(2), p. 146-154, 2010.

SANTOS, J. M., Remediação de sólidos de perfuração via aquecimento por micro-ondas, Uberlândia/MG, UFU, 2014.

SHANG, H.; ROBINSON, J. P.; KINGMAN, S. W.; SNAPE, C. E.; WU, Q. Theoretical study of microwave enhanced thermal decontamination of oil contaminated waste, Chemical Engineering Technology, v. 30(1), p. 121-130, 2007.

SHANG, H., SNAPE, C. E., KINGMAN, S. W., ROBINSON, J. P., Treatment of oilcontaminated drill cuttings by microwave heating in a high-power single-mode cavity, Industrial e Engineering Chemistry research, v. 44(17), p. 6837-6844, 2005.

SHANG, H., SNAPE, C. E., KINGMAN, S. W., ROBINSON, J. P., Microwave treatment of oil-contaminated North Sea drill cuttings in a high power multimode cavity, Separation and Purification Technology, v. 49(1), p. 84-90, 2006. 